\title{
Digital Kymography for the Analysis of the Opening and Closure Intervals of Heart Valves
}

\author{
Sven Friedl ${ }^{1,2}$, Stefan König ${ }^{1}$, Markus Kondruweit ${ }^{2}$, Thomas Wittenberg ${ }^{1}$ \\ ${ }^{1}$ Fraunhofer Institute for Integrated Circuits IIS, Erlangen \\ ${ }^{2}$ University Hospital Erlangen \\ sven.friedl@iis.fraunhofer.de
}

\begin{abstract}
The opening and closure intervals of heart valves are important factors regarding an optimal blood flow. Using endoscopic video recordings of heart valves, digital kymography can be a method to visualize and to describe the opening and closure intervals. Based on an adequate segmentation of the kymograms, a quantitative description becomes available for the opening and closure characteristics. Furthermore, by normalizing the measured results, comparisons of mean heart valve oscillations can be achieved. Here, a comparison of artificial with native heart valves obtained under physiological conditions, indicates clear differences in the opening and closure intervals.
\end{abstract}

\section{Introduction}

The opening and closure intervals of heart valves are important factors regarding an optimal blood flow. In particular for artificial heart valves, an analysis of those intervals is essential to estimate the physiological characteristics and to evaluate the graft as well as the implantation technique. Digital high speed recording of artificial heart valves is a young but promising approach to analyze the movement and behavior of the grafts. For example, Lu et al. [1] as well measured the opening and closure times of different artificial valve types using a high speed camera. Kaminsky et al. [2] evaluated the flow behind the valves using high speed videos and particle image velocimetry. As one major prerequisite for automated measuring analysis is image segmentation, various working groups have proposed approaches for the delineation of the orifice area. Condurache et al. [3] compared threshold-based methods to active contours, whereas Wittenberg et al. [4] focused on active contours to analyze the valve movements. Hahn et al. [5] used different threshold-based methods to apply a frequency analysis to evaluate the flattering of the leaflets. Weiß et al. [6] presented a compact visualization of the movements based on segmentation by an edge-amplified region growing. Nearly all of the digital video recordings of heart valves and the accordant evaluations are restricted to artificial grafts and to experimental setups. Recently alternative methods are evolving, which are allowing to record heart valve movements under physiological conditions [7]. Thus, next to artificial ones, native heart valves also can be examined and the characteristics of both types can be compared with each other. 


\section{Materials and Methods}

\subsection{Endoscopic Video Recordings}

For this pilot study of methods, endoscopic high speed video recordings of four native and one artificial porcine aortic heart valve have been available. The videos have been recorded in explanted pig hearts, which were reanimated and the heart valves were observed with an endoscopic high speed video camera with 2000-4000 frames per second. Having multiple recordings for each valve, depicting approximately two to three systolic phases, in sum, a corpus of 37 phases of native valves and 9 phases of the artificial valve have been analyzed.

\subsection{Digital Kymography}

The principle of digital kymography is to plot extracted or projected image lines along a time axis. Thus, image changes, as movements are, can be determined and visualized in a compact manner. In the field of medical imaging it is applied in different scenarios as CT reconstruction [8] or vocal fold analysis [9].

To generate a kymogram from the high speed video recordings of heart valves, a line segment

$$
l=\{u+r v \mid r \in[0,1]\}
$$

with the vectors $u$ and $(u+v)$ as the end points of $l$ is defined in one initial frame of the video sequence (Fig. 1, top). In each frame $I(t)$ of the complete sequence or a defined subsequence, the pixel under the line segment are extracted to

$$
k(t)=l(I(t))
$$

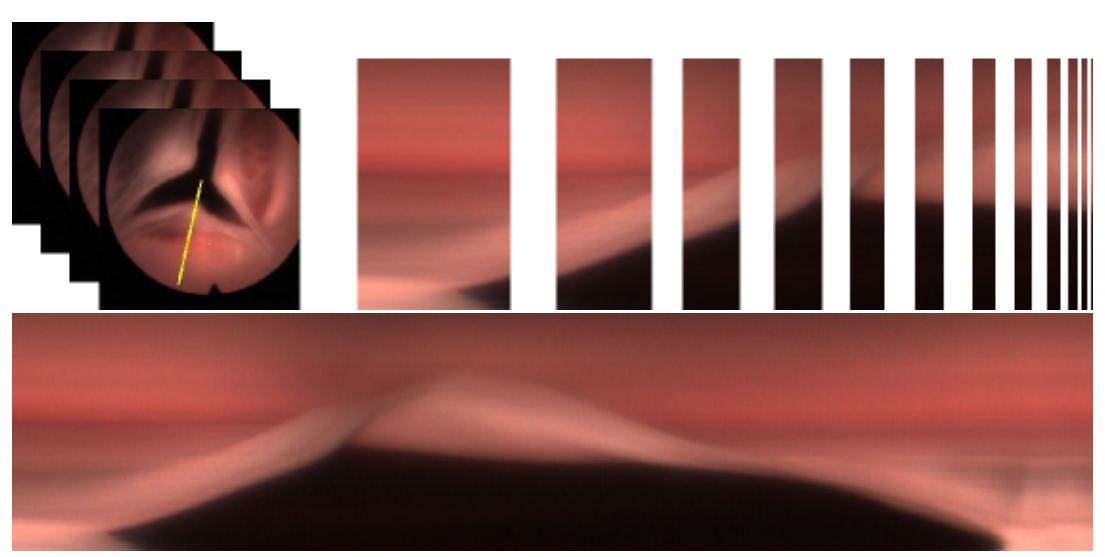

Fig. 1. Generation of digital kymograms from endoscopic heart valve video recordings. Top: definition of the kymogram line and concatenation of the extracted lines; bottom: final digital kymogram of a native aortic heart valve. 
Table 1. Mean interval durations and standard deviations of native and artificial aortic heart valves.

\begin{tabular}{lll}
\hline & Native valves & Artificial valve \\
\hline Opening interval $[\mathrm{ms}]$ & $32.37 \pm 9.99$ & $34.29 \pm 10.24$ \\
Interval of max. orifice $[\mathrm{ms}]$ & $54.57 \pm 28.97$ & $71.61 \pm 9.53$ \\
Closure interval $[\mathrm{ms}]$ & $81.16 \pm 44.52$ & $30.96 \pm 6.50$ \\
\hline
\end{tabular}

The vectors $k(t)$ containing the pixel along the line segment of frame $t$ are then concatenated in horizontal order with

$$
K=\bigcup_{t} k(t)
$$

composing the final kymogram $K$ as can be seen for a native aortic valve in Fig. 1, bottom.

\subsection{Segmentation of Kymograms}

The digital kymogram visualizes the movements of the leaflets in a compact manner. For a more quantitative description, the orifice area within the kymograms can be segmented using simple thresholding. Smaller artifacts can be removed by morphological operations and by regarding only segmented areas with a significant size. Thus the orifice distance during the phases of opening and closing can be extracted from the kymograms. Since the horizontal axis of a kymogram describes the time axis of the video sequence, the orifice opening $d(t)$ of a leaflet can be determined for each discrete time by calculating the vertical length of the segmentation.

With a lack of spatial calibration within the available recordings, normalized orifice distances are computed according to $d^{\prime}=d \cdot \frac{100}{\max (\mathrm{d})}$ to achieve comparable measures. The resulting normalized distances are plotted along a normalized time axis $t^{\prime}$ defined by $t^{\prime}=t \cdot \frac{100}{\sum \Delta t}$ with $\sum \Delta t$ as the sum of the durations of all intervals of one phase.

\section{$3 \quad$ Results}

The 37 phases of native and the 9 phases of the artificial heart valves have been analyzed. Within each of the phases, a digital kymogram has been generated for all of the three leaflets. In the resulting kymograms, specific intervals are defined and the corresponding durations are calculated. For the analysis of the opening and closure of the heart valves, the durations $\Delta t$ of the opening interval, of the phase of maximal orifice and of the closure interval are determined (Fig. 2).

Examining all kymograms of the native, respectively the artificial valves, the mean durations of the different intervals can be determined. Table 1 shows the results for the available recordings. Thus, the differences between the intervals 
and in particular the differences of the intervals between native and artificial valves can be evaluated. In case of the native heart valves, the opening interval is much shorter than the closure interval. These measures match with the known physiology of heart valves. For the artificial heart valves, the interval of maximal orifice is much longer than the opening and closure and the closure interval is significant shorter than in case of native valves.

This fact becomes more noticeable, by observing the normalized measurements of the segmented kymograms. The weighted mean of all normalized kymogram functions respective to time-dependent orifice opening of the native, respectively the artificial valves is computed (Fig. 3). The plots are now containing the movement of a typical mean heart valve and show the quantitative differences between the artificial and the native valves.

\section{Discussion}

Digital kymography enables a fast, convenient and stable analysis of the opening and closure intervals of heart valves. The kymogram allows to estimate the progress of the movements and to measure the durations. With the segmentation of the kymograms and the normalization of the scales, a more quantitative description of typical valve movements is possible. The first results with few recordings indicate clear differences between artificial and native heart valves. Of course, a final conclusion is not yet possible. Due to the complex and costly recording technique, the availability of recordings under physiological conditions is limited. Alternative experimental setups can help to improve and stabilize the methods, but for a clinical study, physiological conditions are essential. Due to the architecture of mechanical caged ball valves, the method of digital kymography as described above is not suitable for those. But for native and artificial biological valves as well as mechanical leaflet valves, digital kymography enables
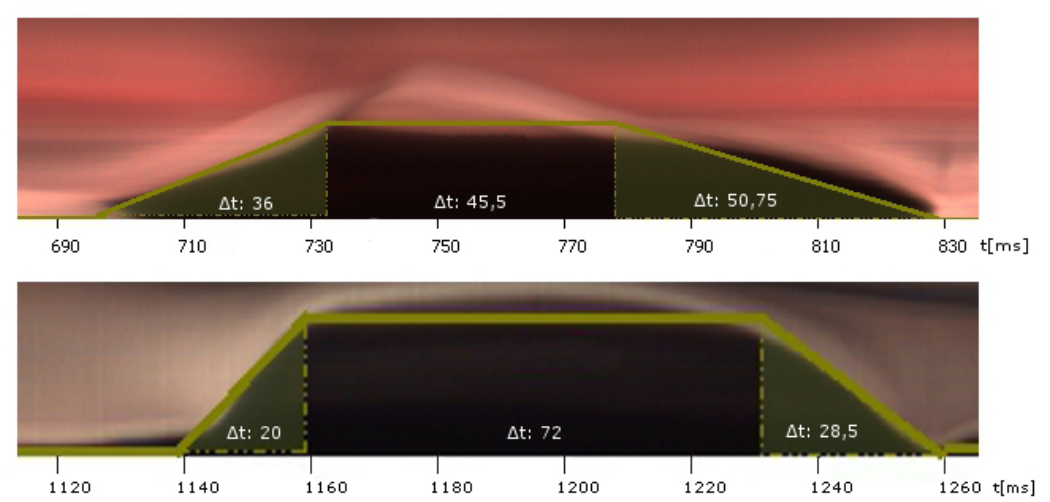

Fig. 2. Kymograms of an aortic heart valve. Comparison of the duration of opening and closure intervals between native (top) and artificial (bottom) valves. 
Fig. 3. Normalized mean movements of native (top) and artificial (bottom) heart valves.
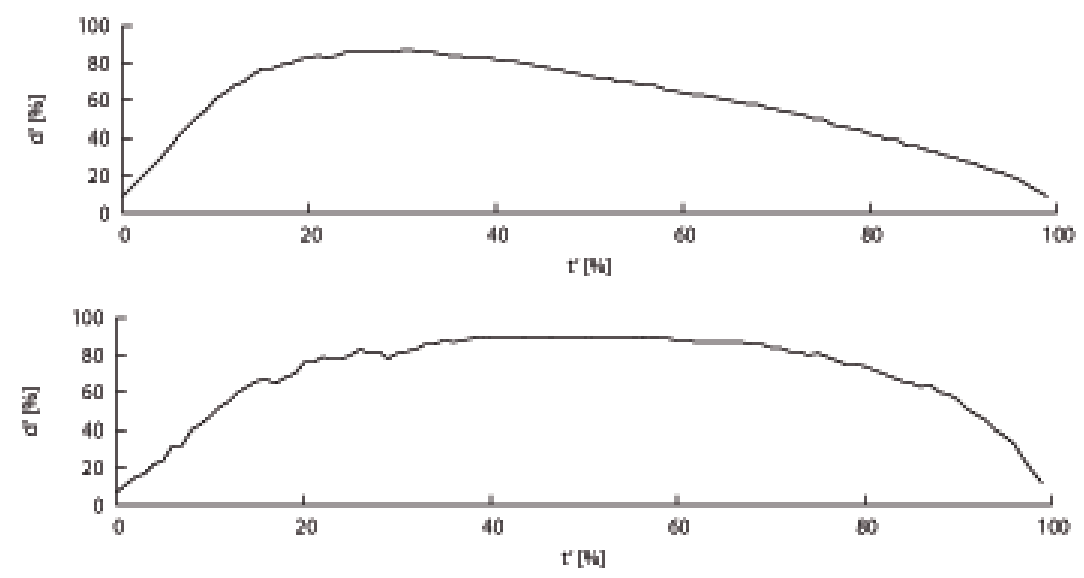

a systematic study of the intervals in different heart valve grafts and a comparison with native ones.

\section{References}

1. Lu PC, Liu JS, Huang RH, et al. The closing behavior of mechanical aortic heart valve prostheses. ASAIO J. 2004;50(4):294-300.

2. Kaminsky R, Kallweit S, Weber H, et al. Stereo High Speed PIV Measurements behind two different artificial heart valves. In: Proc Intl Symp Applic Laser Tech Fluid Mech; 2006.

3. Condurache AP, Hahn T, Hofmann UG, et al. Automatic measuring of quality criteria for heart valves. In: Proc SPIE. vol. 6512; 2007. p. 2Q-1-11.

4. Wittenberg T, Cesnjevar R, Rupp S, et al. High-speed-camera recordings and image sequence analysis of moving heart-valves: experiments and first results. In: Adv Med Eng. vol. 114; 2007. p. 169-74.

5. Hahn T, Condurache AP, Aach T, et al. Automatic in-vitro orifice area determination and fluttering analysis for tricuspid heart valves. In: Proc BVM; 2006. p. $21-25$.

6. Weiß O, Friedl S, Kondruweit M, et al. Aufnahme, Analyse und Visualisierung von Bewegungen nativer Herzklappen in-vitro. In: Proc BVM; 2008. p. 328-32.

7. Kondruweit M, Wittenberg T, Friedl S, et al. Description of a novel ex-vivo imaging and investigation technique to record, analyze and visualize heart valve motion under physiological conditions. In: Proc DGTHG; 2010.

8. Ertel D, Pflederer T, Achenbach S, et al. Validation of a rawdata-based synchronization signal (kymogram) for a phase-correlated cardiac image reconstruction. Eur Radiol. 2008;18(2):253-62.

9. Wittenberg T, Tigges M, Mergell P, et al. Functional imaging of vocal fold vibration: digital multi-slice high-speed-kymography. J Voice. 2000;14(3):422-42. 